\title{
Intramuscular hemangioma of the chest wall: An unusual tumor
}

\author{
Salvatore Griffo, MD, Paolo Stassano, MD, Giuseppe De Luca, MD, Luigi Di Tommaso, MD, Mario Monaco, MD,
} and Sergio Spiezia, MD, Naples, Italy

I ntramuscular hemangiomas (IMHs) are rare benign tumors affecting mainly the muscles of the upper and lower extremities; even rarer are those affecting the chest wall. ${ }^{1,2}$ If they do not cause swelling and pain, they may go unnoticed for many years and an accurate preoperative diagnosis may be difficult. $^{2}$

We report the case of a patient with IMH of the chest wall that went undiagnosed for a long time and was completely resected after 5 years.

\section{Clinical Summary}

A 37-year-old woman was admitted to our hospital with a palpable mass causing cosmetic deformity in the right lateral portion of the chest. This lesion, discovered 5 years earlier and slowly enlarging, had been operated on 3 years previously for suspected lipoma. The clinical history was unremarkable. She was not a smoker, and she denied any history of chest trauma, pain, cough, or respiratory distress. Physical examination revealed a painless soft tissue mass, lacking discrete margins, in the right posterolateral side of the chest and extending into the subscapular area (Figure 1, inset). A chest radiogram revealed an ill-defined soft tissue mass in the right lateral chest wall. A chest computed tomographic (CT) scan showed a right-sided heterogeneous mass extending into the subscapular region, with fatty component, interspersed tubular elements of soft tissue attenuation, and the presence of a calcification (Figure 1). The mass extended into the right serratus and intercostal muscles up to the subscapular region, without osseous abnormalities of the ribs.

Through a small right lateral incision, the mass was completely excised with the surrounding margin of normal muscle. The lesion measured $15 \times 9 \times 4 \mathrm{~cm}$. Macroscopically, the cut surface had a polycystic appearance with some fresh blood and calcification. Microscopically, the tumor revealed a large number of dilated vessels with a single layer of endothelial cells in a skeletal muscle with no signs of atypia or mitosis. There were also areas of fatty tissue, focal calcifications, intravascular thrombus, and fibrous tissue (Figure 2). Histologic examination determined intramuscular cavernous hemangioma.

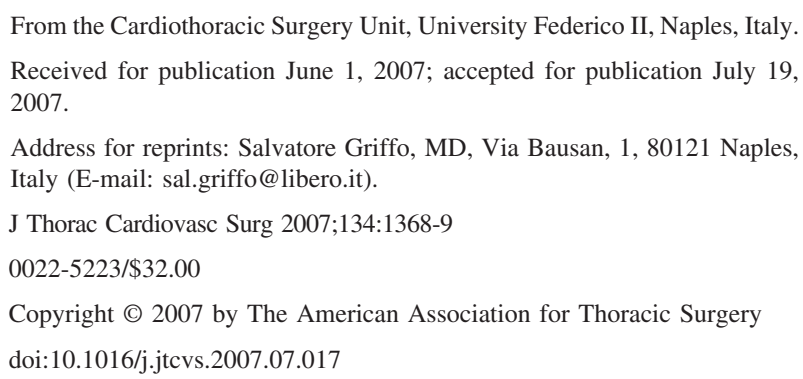

The postoperative course was uneventful, and the patient was discharged home 3 days after the operation. She has been followed up for 2 years without evidence of recurrence.

\section{Discussion}

Chest wall hemangiomas are rare tumors that may originate within the soft tissue or from the ribs. IMHs are infrequent, representing less than $1 \%$ of all hemangiomas, and the localization in the chest wall is even less frequent. ${ }^{1-3}$ They have been classified by Allen and Enzinger ${ }^{4}$ according to the size of the compositional vessels. Approximately $94 \%$ of IMHs occur before the age of 30 years without gender predilection. ${ }^{2,3}$ They are congenital in origin, developing from abnormal embryonic sequestration, although trauma is thought to play an important role as an initiating factor in young patients. ${ }^{2}$ Despite some mitotic activity and infiltration, the tumor is completely benign and never metastatizes. ${ }^{2,3}$

The diagnosis of IMH of the chest wall requires a high index of suspicion. In fact, they lack constitutional symptoms and over $90 \%$ of all intramuscular hemangiomas are misdiagnosed, as was the

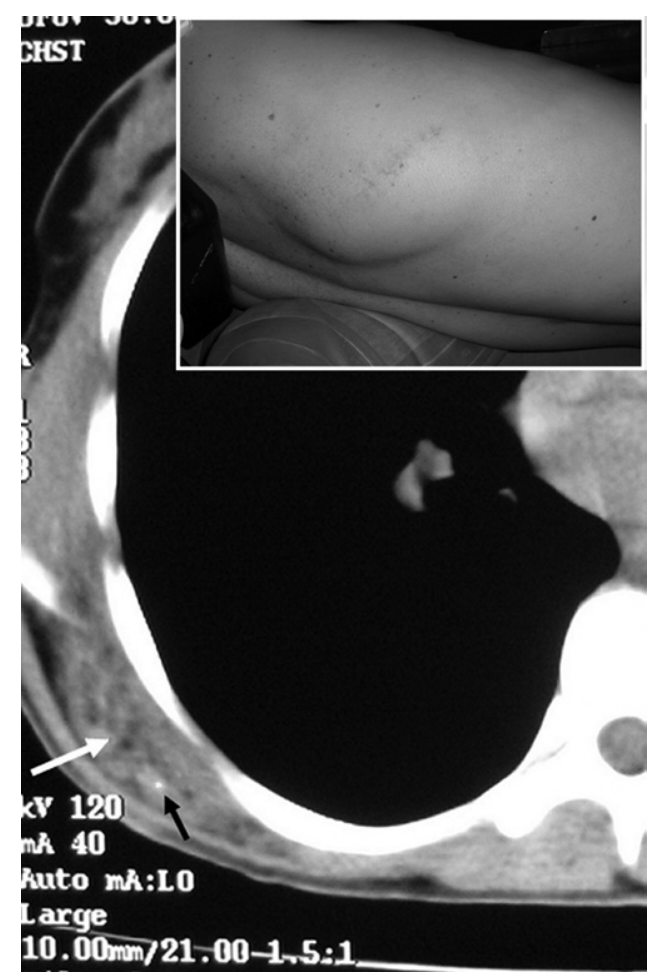

Figure 1. Patient examination reveals the mass located in the right posterolateral chest (inset). CT scan shows the heterogeneous mass (white arrow) with multiple nodular areas of soft tissue attenuation and calcification (black arrow). 


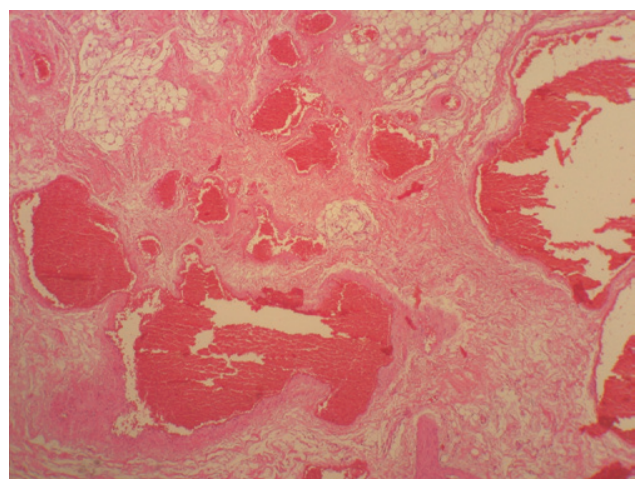

Figure 2. Hematoxylin and eosin-stained section reveals dilated vessels with endothelial cells, thrombus, and areas of fat and fibrous tissue (original magnification $\times 250$ ).

case of our patient. ${ }^{1,3} \mathrm{CT}$ scan is very useful in the evaluation of IMH: it reveals the involvement of adjacent structures and identifies focal calcifications (phleboliths) present in approximately $25 \%$ of cases. ${ }^{3}$ Differential diagnosis includes infection (which was unlikely in our patient because of the 5-year period of time and the lack of general signs), plasmacytoma or primary bone tumor (but there was no osseous involvement), lipoma (but lipoma has no heterogeneous nodular appearance), liposarcoma (but the phleboliths are unusual in liposarcoma), neurogenic tumor (which is located near the spine), desmoid tumor (which lacks phleboliths), and elastofibroma dorsi (which have a more compact tissue and usually are bilateral). ${ }^{1}$

In any case, the definitive diagnosis is made by histologic study of the surgical and/or biopsy specimen.

Complete surgical excision with clear-cut margins is the best therapeutic approach, although every patient with IMH should be treated individually with consideration given to tumor location, surgical accessibility, depth of invasion, patient's age, and cosmetic factors. Radiotherapy, cryotherapy, embolization, electrocoagulation, and injection of sclerosing agents may be beneficial in case of impractical and/or only partial surgical exeresis. ${ }^{2,3}$ A local recurrence in up to $18 \%$ of patients after surgical resection has been reported. ${ }^{5}$

In conclusion, the rarity of IMH of the chest wall may render an accurate preoperative diagnosis difficult. A high index of suspicion is required, and complete surgical excision is mandatory.

\section{References}

1. Ly JQ, Sanders TG. Case 65: hemangioma of the chest wall. Radiology. 2003;229:726-9.

2. Sherman JA, Davies HT. Intramuscular hemangioma of the temporalis muscle. J Oral Maxillofac Surg. 2001;59:207-10.

3. Wild TA, Raab P, Krauspe R. Hemangioma of skeletal muscle. Arch Orthop Trauma Surg. 2000;120:139-43.

4. Allen PW, Enzinger FM. Hemangioma of skeletal muscle: an analysis of 89 cases. Cancer. 1972;29:8-22.

5. Cohen AJ, Youkey JR, Claggett GP, Huggins M, Nadalo L, d'Avis JC. Intramuscular hemangioma. JAMA. 1983;249:2680-2.

\title{
Use of an apical heart suction device to expose the left pulmonary veins during transsternal pneumonectomy
}

\author{
Federico Venuta, MD, a Giuseppe Mazzesi, MD, ${ }^{\mathrm{b}}$ Federico Francioni, MD, ${ }^{\mathrm{a}}$ Marco Anile, MD, ${ }^{\text {a }}$ Mario Di Stasio, MD, ${ }^{\mathrm{a}}$
} Valeria Liparulo, MD, ${ }^{a}$ Chiara Ricella, MD, a and Giorgio Furio Coloni, MD, ${ }^{\text {a }}$ Rome, Italy

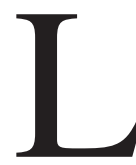

ung cancer operations are usually performed through a standard lateral or posterolateral thoracotomy. However, there are some difficult cases with an extended proximal invasion of the pulmonary vessels (artery and/or veins) ${ }^{1}$ or the carina ${ }^{2}$ that may require a median

From the Departments of Thoracic Surgery ${ }^{\mathrm{a}}$ and Cardiac Surgery, ${ }^{\mathrm{b}}$ University of Rome "La Sapienza," Rome, Italy.

Received for publication Jan 18, 2007; revisions received April 10, 2007; accepted for publication April 20, 2007.

Address for reprints: Federico Venuta, MD, Cattedra di Chirurgia Toracica, Università di Roma "La Sapienza," Policlinico Umberto I, V.le del Policlinico, 00100 Rome, Italy (E-mail: sofed@libero.it).

J Thorac Cardiovasc Surg 2007;134:1369-71

$0022-5223 / \$ 32.00$

Copyright $\odot 2007$ by The American Association for Thoracic Surgery doi:10.1016/j.jtcvs.2007.04.071 sternotomy approach. This incision allows optimal control of the root of the vessels, the ideal exposure for complex vascular reconstructions, and the institution of cardiopulmonary bypass $(\mathrm{CPB})$ with central cannulation if required. The latter may be indicated either to reconstruct a vessel invaded too proximally by the tumor or to address emergency situations. It could also be required to improve exposure of the posterior aspect of the heart and in particular the left atrium. It helps to decompress the cardiac chambers when the heart cannot be vertically lifted by hand retraction. In these situations, a median and upward retraction of the heart could lead to poor diastolic filling, hypotension, and hemodynamic instability. The use of the bypass machine has been shown to activate the inflammatory response, ${ }^{3}$ require full systemic heparinization, and increase the need for blood transfusions, ${ }^{4}$ especially during more complex operations. For these reasons, it should be avoided whenever possible during lung surgery. 